attribute to increased risk of cancers can be genomic instability and impaired DNA repair.

Objectives: The aim of the study was to assess the processes of endogenous and exogenous DNA damage and its repair in patients with IIM as compared to healthy controls.

Methods: The study included 10 patients ( 9 men and 1 woman, mean age 46,6 $\pm 16,5$ ) with idiopathic inflammatory myopathies (dermatomyositis or polymyositis) as well as 7 healthy control individuals ( 4 men and 3 women, mean age $33,8 \pm 9,8$ ). DNA damage and repair were investigated by the comet assay. To perform the comet assay human peripheral blood mononuclear cells (PBMCs) were isolated and incubated with tert-butyl hydroperoxide (t-BOOH) or bleomycin. Both compounds are common DNA damaging agents - $t-B O O H$ induces oxidative DNA lesions whereas bleomycin induces also DNA double strand breaks (DSBs). To test the DNA repair capability, PBMCs were allowed to recover for 2 hour. The level of endogenous DNA lesions was also investigated.

Results: The levels of endogenous DNA damage were not statistically significantly different between tested groups (IIM-3,3 $\pm 3,6 \%$ vs $3,2 \pm 3,8 \%$ in control; $\mathrm{p}=0,648$ ). The extent of the DNA damage induced by bleomycin (IIM-23,3 $\pm 19,5 \%$ vs $9,8 \pm 5,9 \%$ in control) as well as oxidative stress (IIM-14,7 $\pm 16,2 \%$ vs $10,4 \pm 7,4 \%$ in control) was significantly higher in PBMS derived from IIM patients than in healthy counterparts $(p<0,001)$. Kinetic curves of DNA repair are different but the background mechanism underlying observed differences in the repair curve between healthy subjects and patients need to be evaluated further.

Conclusions: Understanding the etiology of this phenomena in these diseases may provide insight into disease pathogenesis and explain the increased susceptibility of patients to malignancies. Finding the patients with increased DNA instability could potentially serve as a biomarker and indicate the group of patients who should be carefully screened for neoplasmatic disorder.

Disclosure of Interest: None declared

DOI: 10.1136/annrheumdis-2018-eular.5973

\section{AB0011 EVALUATION OF SALIVARY MIRNAS IN PATIENTS AFFECTED BY SJÖGREN'S SYNDROME AND CORRELATION WITH CLINICAL AND ULTRASONOGRAPHIC OUTCOMES}

R. Talotta ${ }^{1}$, M. Biasin ${ }^{2}$, S. Bongiovanni ${ }^{1}$, V. Mercurio ${ }^{2}$, C. Vittori ${ }^{2}$, L. Boccassini ${ }^{1}$, F. Rigamonti ${ }^{1}$, A. Batticciotto ${ }^{1}$, F. Atzeni ${ }^{3}$, D. Trabattoni ${ }^{2}$, P. Sarzi-Puttini ${ }^{1}$.

${ }^{1}$ Rheumatology Unit, ${ }^{2}$ Biomedical and Clinical Sciences, University Hospital "Luigi

Sacco", Milan; ${ }^{3}$ Rheumatology Unit, AOU "G.MARTINO", Messina, Italy

Background: It has been demonstrated that miRNAs expressed in PBMCs and salivary glands of patients affected by Sjögren's Sydrome (SS) could be involved in the epigenetic control of the disease.

Objectives: We aimed to compare the concentration of miRNA-146a/b, 16, 1792 cluster and 181a in salivary and plasmatic samples collected from SS patients and healthy controls and to evaluate the associations with clinical, laboratory and ultrasound findings.

Methods: We collected plasmatic and salivary samples from 28 patients (27 females, mean age $64.4 \pm 10.1$ years, mean disease duration $10.7 \pm 6.9$ years) affected by primitive SS according to ACR 2012 and/or 2016 criteria and 23 matched healthy controls. In the group of patients, the following data were recorded: ESSDAI and ESSPRI scores, anti-SSA and anti-SSB status and laboratory data, Schirmer's test, ultrasound scores of the four major salivary glands according to Cornec et al. and concomitant treatments. The following miRNA were extracted, retro-transcribed and quantified: $\operatorname{miR} 16-5 \mathrm{p}, \operatorname{miR} 17-5 \mathrm{p}, \operatorname{miR} 18 \mathrm{a}-5 \mathrm{p}, \operatorname{miR} 19 \mathrm{a}-5 \mathrm{p}$, miR19b-1-5 p, miR20a, miR92-5 p, miR146a-5p, miR146b-5p, miR181a-5p. Results: The concentration of miRNAs evaluated in plasma and saliva did not significantly match both in patients and in controls, underlining a different modulation in their expression according to the corporeal district. In patients and controls miRNAs16, 17 and 20 were hyper-expressed and miRNA146b and 181 hypoexpressed in salivary samples compared to plasmatic ones. Salivary miRNAs 18a and 146a were hyper-expressed in patients and hypo-expressed in controls, when compared to plasmatic concentrations.

Comparing salivary and plasmatic patients' miRNAs concentrations to those of healthy subjects, plasmatic miRNA16 and 181a were significantly more expressed in patients than in controls (two-tailed Wilcoxon test and Student's T test for unpaired samples).

In SS patients, salivary miRNA 181 and 146a were significantly increased in older subjects ( $p=0.01$ and $p=0.04$ respectively). Spearman's correlation revealed that salivary miRNA146b was significantly hyper-expressed in patients with worse ESSPRI scores $(p=0.02)$; on the contrary, salivary miRNA17, 146b and plasmatic miRNA17 were reduced in patients with higher scores at ultrasound evaluation $(p=0.01, p=0.01$ and $p=0.04$ respectively). Plasmatic concentration of miRNA18a was increased in patients with less lachrymal production at Schirmer's test $(p=0.01)$ and plasmatic concentration of miRNA17 was reduced in patients with higher ESR values $(p=0.01)$. Salivary miRNA18a was significantly increased in patients with anti-La/SSB ( $\mathrm{p}=0.04$; Mann-Whitney $U$ test for unpaired samples). Salivary and plasmatic miRNAs did not correlate with disease duration and concomitant therapies.

Conclusions: Our data show that the expression of salivary miRNAs 17, 18a and $146 \mathrm{~b}$ may be altered in SS patients and associated with worse ultrasound and ESSPRI scores and anti-La/SSB positivity.

Disclosure of Interest: None declared

DOI: 10.1136/annrheumdis-2018-eular.4876

\section{AB0012 ALLELE AND GENOTYPE FREQUENCY OF SOME GENE POLYMORPHISMS DIFFERS IN VARIOUS JUVENILE IDIOPATHIC ARTHRITIS SUBTYPES}

H.A. Yatskiu' ${ }^{1}$, N.V. Nikitchenko ${ }^{1}$, N.V. Savina ${ }^{1}$, T.D. Kuzhir ${ }^{1}$, A.M. Tchitchko ${ }^{2}$, I. D. Chyzheuskaya ${ }^{3}$, N.L. Bilskaya ${ }^{4}$, M.G. Myslivets ${ }^{5}$, A.V. Sukalo ${ }^{2}, \underline{\text { R. }}$

1. Goncharova ${ }^{1}{ }^{1}$ Institute of Genetics and Cytology of NAS of Belarus; ${ }^{2}$ Belarusian State Medical University; ${ }^{3}$ th City Children's Clinical Hospital, Minsk; ${ }^{4}$ Gomel Regional Children's Clinical Hospital, Gomel; ${ }^{5}$ Grodno Regional Children's Clinical Hospital, Grodno, Belarus

Background: In spite of the fact that chronic arthritis has been the core of paediatric rheumatology, etiology and pathogenesis of juvenile idiopathic arthritis (JIA) are still unclear. It is important to ascertain genetic nature of such a heterogeneous disease.

Objectives: The aim of the study was to assess the role of some SNPs of six genes implicated in immune and inflammatory responses: TNF $\alpha$ (rs1800629, rs361525), PTPN22 (rs2476601), MIF (rs755622, rs5844572), TRAF/C5 (rs3761847), CTLA4 (rs5742909, rs231775), STAT4 (rs7574865); as well as homozygous deletions of xenobiotic biotransformation genes GSTT1 and GSTM1.

Methods: 206 patients diagnosed with JIA (mean age $8.87 \pm 4.92$ ), and 218 hospital controls with no signs of autoimmune or inflammatory diseases (mean age13.88 \pm 2.72 ) were recruited for the study. The JIA patients were divided into subgroups according to IIAR classification criteria; the majority of them (125 patients) had oligoarthritis, 42 children developed RF-negative polyarthritis and 27 patients were diagnosed with systemic arthritis. Genomic DNA was extracted from peripheral blood samples by means of the phenol-chloroform method. SNPs were genotyped using PCR-RFLP, Real-Time PCR or fragmental analysis.

Results: The allele frequencies for all SNPs in the hospital control group were similar to those in European populations. The allele and genotype frequency distribution for all SNPs was identical in patients and controls. However, when comparing distinct subtypes of JIA, STAT4 polymorphism demonstrated higher frequencies of minor T allele $(30.5 \%$ vs. $17.8 \%, \mathrm{p}=0.01, \mathrm{OR}=2.03,95 \% \mathrm{Cl}[1.14$ 3.6] and $\mathrm{G} / \mathrm{T}$ genotype (46.3\% vs. $25.6 \%, \mathrm{p}=0.03$, OR=2.51, 95\%[1.2-5.24]) in RF-negative polyarthritis than in oligoarthritis. On the contrary, $\triangle$ GSTT1 appeared to prevail in oligoarthritis $(23.7 \%$ vs. $7.9 \%$ in RF-negative polyarthritis, $p=0.03$, $\mathrm{OR}=3.6,95 \% \mathrm{Cl}[1.03-12.7])$. As to systemic arthritis, it was shown, that minor $\mathrm{G}$ allele of TRAF/C5 was more frequent in comparison with oligoarthritis $(p=0.037$, $\mathrm{OR}=2.43,95 \% \mathrm{Cl}[1.05-5.6])$.

Conclusions: The results obtained can be considered as evidence for distinct genetic nature of the different JIA subtypes.

Disclosure of Interest: None declared

DOI: 10.1136/annrheumdis-2018-eular.5087

\section{AB0013 EXPRESSION LEVELS OF MIR-124 IN THE PLASMA OF RHEUMATOID ARTHRITIS PATIENTS}

R. Shumnalieva ${ }^{1}$, D. Kachakova ${ }^{2}$, V. Shoumnalieva-Ivanova ${ }^{3}$, P. Miteva ${ }^{4}$, R. Kaneva ${ }^{2}$, Z. Kolarov ${ }^{1}$, S. Monov ${ }^{1} .{ }^{1}$ Department of Internal Medicine, Clinic of rheumatology, ${ }^{2}$ Molecular Medicine Center, Department of Medical Chemistry and Biochemistry, Medical University - Sofia, ${ }^{3}$ University Hospital "Alexandrovska", Medical University - Sofia, Bulgaria, ${ }^{4}$ University Hospital "Prof. Ivan Mitev", Medical University - Sofia, Bulgaria, Sofia, Bulgaria

Background: Micro-ribonucleic acids (microRNAs) comprise a class of small non-coding RNAs that regulate gene expression on post transcriptional level. Levels of miR-124 have been found to be decreased in rheumatoid arthritis (RA) synoviocytes and in vivo studies have shown that treatment with pre-miR-124 suppresses the progression of joint damage ${ }^{1}$

Objectives: To evaluate the expression levels of miR-124-3 $p$ in plasma of RA patients and to determine its possible role as biomarker for diagnosis and disease activity.

Methods: 34 RA patients according to the 1987 ACR criteria were included in the study. Expression levels of miR-124-3 $p$ in the plasma were determined by PCR 
(SYBR Green technology). $2^{-\Delta \Delta C t}$ method was used for analysis. 10 healthy donors were used as controls.

Results: Expression levels of miR-124-3 p were upregulated in the plasma of 33 $(97.06 \%)$ of the patients when compared to controls. Receiver operating characteristic curve analysis was conducted in order to evaluate the diagnostic accuracy of the expression levels of the studied miRNA in the plasma. Area under the curve for miR-124-3 $p$ was $0.879(95 \% \mathrm{Cl}=0.740-1.00), p=0.303 \times 10-3$. When the relative quantification $(\mathrm{RQ})$ cut value was 2.89 , the sensitivity was $91.2 \%$ and the specificity was $80 \%$. Levels of miR-124-3 $p$ in plasma correlated with the diagnosis $(p=0.106 \times 10-3)$, with markers of inflammation $-E S R(p=0.0497)$ and CRP $(p=0.047)$ as well as with the immunological activity - the presence of RF $(p=0.007), R F \operatorname{lgM}(p=0.004), R F \operatorname{lgG}(p=0.004), R F \operatorname{IgA}(p=0.005)$ and anti-CCP antibodies in the serum $(\mathrm{p}=0.025)$.

Conclusions: In contrary to the literature data that report levels of miR-124 to be decreased in RA synovial fibroblasts we found increased expression of miR-124$3 p$ in the plasma of RA patients which might reflect the pathophysiological response to the inflammation, the effect of the treatment regimen or the presence of miR-124a gene promotor hypermethylation in the synovial tissue which might downregulate miR-124 locally. ${ }^{12}$ To our knowledge this is the first study to evaluate the diagnostic accuracy of plasma levels of miR-124 in RA patients as well as the possibility of using miR-124 as biomarker for disease activity but larger set is needed to confirm these results in the clinical practice.

\section{REFERENCES:}

[1] Nakamachi $Y$, et al. MicroRNA-124a is a key regulator of proliferation and monocyte chemoattractant protein 1 secretion in fibroblast-like synoviocytes from patients with rheumatoid arthritis, Arthritis Rheum 2009;60 (5):1294-1304

[2] Zhou Q, et al. Research of the Methylation Status of miR-124a Gene Promoter among Rheumatoid Arthritis Patients. Clin Dev Immunol 2013 (2013):524204

Acknowledgements: The study was supported by Grant 76/2016 funded by Medical University - Sofia, Bulgaria.

Disclosure of Interest: None declared

DOI: 10.1136/annrheumdis-2018-eular.5851

\section{AB0014 GENETIC INFLUENCE OF DIFFERENT MEASURE FOR TUMOUR NECROSIS FACTOR INHIBITORS RESPONSE IN RHEUMATOID ARTHRITIS}

S. Bang ${ }^{1}$, Y. Park ${ }^{2}$, K. Kim ${ }^{3}$, Y.B. Joo ${ }^{4}$, S.-K. Cho ${ }^{2}$, C.-B. Choi ${ }^{2}$, Y.-K. Sung ${ }^{2}$, T.H. Kim ${ }^{2}$, J.-B. Jun ${ }^{2}$, D.-H. Yoo ${ }^{2}$, H.-S. Lee ${ }^{1}$, S.-C. Bae ${ }^{2} .{ }^{1}$ Rheumatology, Hanyang University Hospital for Rheumatic Diseases, Guri; ${ }^{2}$ Rheumatology, Hanyang University Hospital for Rheumatic Diseases; ${ }^{3}$ Biology, Kyung Hee University, Seoul; ${ }^{4}$ Rheumatology, St. Vincent's Hospital, Catholic University, Suwon, Korea, Republic of Ireland

Background: The genetic studies of tumour necrosis factor inhibitors (TNFi) response in patients with rheumatoid arthritis (RA) have largely relied on the changes in complex disease scores as a measure of treatment response. It is expected that genetic architecture of such complex score is heterogeneous and not very suitable for pharmacogenetic studies.

Objectives: We aimed to select the most optimal phenotype for TNFi response using heritability estimates using genome-wide association studies (GWAS) in the Korean population.

Methods: Disease Activity Scores based on 28 joint counts (DAS28) and Clinical Disease Activity Index (CDAl) were assessed at baseline, and after 6 months in 370 Korean RA patients who started TNFi due to moderate or high disease activity. Genotypes were generated on the Illumina HumanOmni2.5Exome array (2.5 million variants) in TNFi-treated Korean patients with RA. We estimated heritability using a linear mixed-modelling approach (GCTA) for the TNFi drugresponse phenotype $\triangle \mathrm{DAS} 28, \triangle \mathrm{CDAl}$ and its separate components, such as $\Delta$ swollen joint count (SJC), $\Delta$ tender joint count (TJC), $\Delta$ erythrocyte sedimentation rate (ESR), $\Delta$ visual-analogue scale of general health (VAS-GH) and $\Delta$ provider global assessment of disease activity (PrGA). Furthermore, a multivariate GWAS approach was implemented, analysing separate DAS28 and CDAI components simultaneously

Results: The highest heritability estimates were found for $\triangle \operatorname{PrGA}\left(h^{2}=0.76\right)$ and $\Delta$ TJC $\left(h^{2}=0.73\right)$; lower heritability was found for $\triangle \mathrm{DAS} 28\left(\mathrm{~h}^{2}=0.32\right)$ with estimates for $\Delta$ ESR $\left(h^{2}=0.66\right), \Delta S J C\left(h^{2}=0.62\right), \Delta \operatorname{CDAl}\left(h^{2}=0.60\right)$ and $\Delta$ VAS-GH $\left(h^{2}=0.53\right)$ (all p-value<0.005)

Conclusions: Our results indicate that multiple SNPs together explain a substantial portion of the variation in change in provider global assessment of disease activity in TNFi-treated patients with RA. In conclusion, optimal phenotype based on heritability suggests the use of changes in clinical disease activity index (CDAl) including provider global assessment than DAS28 in pharmacogenetic study.

Disclosure of Interest: None declared

DOI: 10.1136/annrheumdis-2018-eular.5286

\section{AB0015 THE EFFECT OF RARE CODING VARIANTS ON RESPONSE OF TNF INHIBITORS TREATMENT IN RHEUMATOID ARTHRITIS}

S. Bang ${ }^{1}$, Y. Park ${ }^{2}$, H.-H. Kwon ${ }^{1}$, H. Yoo ${ }^{1}$, H.-S. Lee ${ }^{1}$, S.-C. Bae ${ }^{2} .{ }^{1}$ Rheumatology, Hanyang University Hospital for Rheumatic Diseases, Guri, ${ }^{2}$ Rheumatology, Hanyang University Hospital for Rheumatic Diseases, Seoul, Korea, Republic of Ireland

Background: Although pharmacogenetic studies of TNF inhibitors (TNFi) response presented the estimates of high heritability, only few loci with suggestive weak common association as biomarkers for TNFi response have been identified. Objectives: We aimed to identify novel functional rare variants associated with response to etanercept using targeted exon sequencing in Korea.

Methods: Disease activity scores were assessed at baseline and after 6 months in 156 Korean RA patients who started etanercept due to moderate or high disease activity. We analysed targeted exon sequencing data of 399 genes selected from a multifaceted approach. We conducted a single-marker association test $(\mathrm{MAF} \geq 1 \%)$ and a gene-based analysis [optimal sequence kernel association test (SKAT-O)] of rare variants (MAF $<1 \%$ ). In addition, we performed gene set analyses of TNF pathway genes.

Results: We identified that clinical factors seem to influence the therapeutic good response of etanercept including male, high disease activity score at baseline, BMI. After stringent quality control, we analysed 14024 variants of 399 genes in 156 RA patients. We identified two novel significant functional SNPs [rs16942564, rs61734378 (exon of AKAP13)] associated with response to etanercept, surpassing study-wide significant threshold $\left(p<3.0 \times 10^{-5}\right)$ in single variant association tests. Using a gene-based approach, we found two genes with nominal burden signals $(p<0.001)$ which did not reach study-wide significance. In the gene set enrichment test, we found no evidence for enrichment of association at genes involved in the TNF pathway.

Conclusions: We were unable to identify rare coding variants with large effect of 399 targeted genes. Our study suggests that rare coding variants of RA risk associated genes do not contribute to heritability of response to etanercept therapy.

Disclosure of Interest: None declared

DOI: 10.1136/annrheumdis-2018-eular.6862

\section{$\mathrm{AB} 0016$ \\ CHROMATIN LOCALIZATION OF SURVIVIN IN CD4+ T- CELLS OF PATIENTS WITH RHEUMATOID ARTHRITIS}

S. Andres ${ }^{1}$, K.M. Andersson ${ }^{2}$, A. Damdimopoulos ${ }^{3}$, G. Katona ${ }^{1}$, M.I. Bokarewa ${ }^{2,4}$ ${ }^{1}$ Department of Chemistry and Molecular Biology, ${ }^{2}$ Department of Rheumatology and Inflammation Research, University of Gothenburg, Gothenburg; ${ }^{3}$ Department of Biosciences and Nutrition, Karolinska Institutet, Solna; ${ }^{4}$ Sahlgrenska University Hospital, Gothenburg, Sweden

Background: Oncoprotein survivin emerged as an important player in the pathogenesis of rheumatoid arthritis (RA). Results of genome-wide study suggest that survivin may take part in transcription stimulation of the RA-specific genes.

Objectives: To identify and describe survivin-dependent differences in transcription pattern between $\mathrm{CD} 4^{+} \mathrm{T}$-cells of RA patients and healthy subjects focusing in particular on a subset of genes involved in maturation of Th1 and Th17 cells.

Methods: $\mathrm{CD} 4^{+}$T-cells were isolated from PBMC of 3 RA patients and 5 nonsmoking and 2 smoking healthy controls using a positive selection and activated by Pam3cys+Concanavalin A+LPS. Chromatin immunoprecipitation (ChIP) was done using rabbit polyclonal anti-Survivin, purified DNA was prepared into libraries using ThruPLEX (Rubicon) and sequenced using Hiseq 2000 (Illumina). Resulting fastq sequencing files were mapped to the human reference genome (hg38) using the STAR aligner. Peaks were associated with the closest transcription start site. Enriched peak regions $(p<10-5)$ were identified in survivin-ChIP samples above background ("input") using the Homer software. The peaks were analysed using gene ontology (GO) technique as implemented in GOrilla and GSEA software. The genes, scored high in RA and not present/low in any controls or vice versa were identified. The enriched GO groups were searched for presence of Th1/Th17 regulating genes.

Results: We identified 11145 survivin-bound chromatin sequencies. Out of them, GO technique indicated 770 genes in RA samples (7.3\%) and 766 genes in healthy controls (19.5\%) which were annotated and enriched (>log-5) in GO 